\title{
Introduction to the Special Issue on the Cross-Media Analysis for Visual Question Answering
}

\begin{abstract}
Visual Question Answering (VQA) is a recent hot topic in multimedia analysis, computer vision, natural language processing, and even a broad perspective of artificial intelligence, which has attracted a large amount of interest from the deep learning, computer vision, and natural language processing communities. Given an image (or a video clip) and a question in natural language, VQA requires grounding textural concepts to visual elements to infer the correct answer. The challenge lies in that, in most cases, it requires reasoning over the connection between visual content and languages as well as external knowledge. Towards general applications, besides the understanding of visual content, potential abilities of VQA largely come from the leveraging of different kinds of data (e.g., visual, audio, and text, etc.) across multiple sources (e.g., social-media sites, surveillance videos, and Wikipedia, etc.) for knowledge discovering and QA reasoning, which is recognized as cross-media analysis in multimedia scope.

From the above background, this special issue focuses on new theories and algorithms for visual question answering via cross-media analysis, as well as their applications in the practice of human-computer interaction, multimedia search, visual description for the blind, incident reports for surveillance, seeing chat bots, or even robotic intelligence. Though booming recently, VQA still remains in its infancy, as there are so many challenges. First, to boost the research of VQA, several benchmark datasets have been released. On one hand, more benchmarks solicit larger advances in VQA research; on the other hand, the proposed methods are always specific to certain datasets that lack generalization ability to some extent, as different benchmarks may have different characteristics of their own. Second, though reasoning ability is always claimed, most "reasoning" simply uncovers latent connections between visual elements and textual/semantic facts during the training on a large number of images/videos annotated with QA pairs. As reasoning depends on knowledge, it remains implicit how to appropriately and effectively incorporate the knowledgebased reasoning into the data-driven learning framework of VQA, e.g., an end-to-end deep neural network. Third, as VQA involves multiple modalities of data, towards a broad perspective of applications, cross-media knowledge discovering and reasoning has yet to be well exploited in existing VQA research.
\end{abstract}

This special issue focuses on VQA techniques and its applications. We expect that it will provide an integrated and synthesized view of the state-of-the-art as well as best current practices. Submissions were from an open call for papers that underwent a thorough selection process. Eight papers were accepted for this issue. They can be roughly divided into three categories: (1) image question answering, (2) video question answering, and (3) other applications related to visual question answering.

The first category of this special issue contains three papers that focus on generating answers for the questions over a single image. In the first paper, entitled "Semantic Concept Network and Deep Walk Based Visual Question Answering,” Li et al. propose a semantic concept network and deep-walk-based VQA model. They claim that co-occurrence patterns of concepts can enhance semantic representation, and semantic concepts have complex interrelations and the relationships are similar to a network. Then, Word Activation Forces (WAFs) is adopted to construct a semantic

(C) 2019 Copyright held by the owner/author(s).

1551-6857/2019/07-ART48

https://doi.org/10.1145/3337985 
concept network, and deep walk is used to mine the co-occurrence patterns of semantic concepts. Experiments are conducted to assess the visual question answering and to confirm the advantages of the proposed method. The second paper, entitled "BTDP: Toward Sparse Fusion with Block Term Decomposition Pooling for Visual Question Answering,” by Fang et al., proposes the Block Term Decomposition Pooling (BTDP) architecture as an enhancement of the existing bilinear pooling models to improve VQA models. Moreover, they demonstrate that introducing sparsity into bilinear pooling can significantly increase the performance of feature fusion. Various experiments verify that BTDP is very flexible and it outperforms current bilinear methods. In the third paper, titled "Multi-source Multi-level Attention for Visual Question Answering," Yu et al. introduce a scheme named multi-source multi-level attention network (MSMLAN). The scheme improves VQA performs by: (1) introducing attention network to external knowledge domain, including context-aware visual attention, concept attention, and knowledge attention; and (2) using 2D RNN to encode contextual information of image regions. Experiments on VQA datasets demonstrate the effectiveness of the scheme.

Different from the first category, which generates objective descriptions for single images, the second part contains two papers focusing on producing an accurate answer for a pair consisting of a video clip and a question. Considering the inherent temporal structure in video, approaches of video question answering vary from Image QA schemes. The paper "Video Question Answering via Knowledge-based Progressive Spatial-Temporal Attention Network" introduces a knowledgebased progressive spatial-temporal attention network (K-PSTANet) to tackle the video question answering, in which the spatial and temporal dimension information of video content are considered. By leveraging external knowledge together with questions to guide progressive video attention, K-PSTANet can learn fine-grained joint video representation. The authors construct a large-scale real video dataset to verify the superiority of the method. In the paper "Spatiotemporal-Textual Co-attention Network for Video Question Answering," authors worked towards refining video question answering results by precise joint understanding of videos and questions. They propose a new Spatiotemporal-Textual Co-Attention Network (STCANet) that simultaneously learns spatially and temporally visual attention on videos and textual attention of questions. The proposed approach is applied to two challenging Video QA datasets and indeed achieves better performance.

The last three papers focus on visual story generation, image captioning, and cross-modality retrieval. In their paper entitled "Show, Reward and Tell: Adversarial Visual Story Generation," Tang et al. present an Attribute-based Hierarchical Generative model with Reinforcement Learning and adversarial training (AHGRL) to address the problem of generating narrative creations from ordered photo streams. They propose an attribute-based hierarchical generative model to generate narrative paragraphs and a reinforcement learning framework to update the story generator. By employing an adversarial training strategy during the generator and the discriminator, the quality of the generated narrative paragraphs is improved. The paper "Image Captioning by Asking Questions" introduces a VQA-improved image-captioning method, in which a VQA model was incorporated to enhance an image-captioning model. It works by fusing the VQA-grounded feature and the attended visual feature extracted from the image. The method consists of three steps of VQA-grounded feature representation extraction, VQA-grounded feature and attended visual feature fusion, and language modeling. Comprehensive evaluations of image captioning are conducted on the benchmarks, and the performance of their proposed framework is superior to the state-of-the-art methods. "Cross-Modality Retrieval by Joint Correlation Learning," by Wang et al., presents a cross-modal learning model with joint loss optimization to handle the cross-modality retrieval. They claim that the relationships between the unpaired data is significant, besides the similarity of the paired image-sentence. Therefore, they design a correlation loss function consisting of three views, i.e., the intra-correlation from the visual embedding, the relevant similarity of 
image-sentence pairs, and the inter-correlation from both positive and negative image-sentence pairs. Thus, both positive and negative samples are utilized to optimize in the batch. Experiments on real surveillance videos demonstrate its superiority.

In conclusion, the papers in this special issue cover the techniques addressing different challenges in visual question answering. We believe this issue will benefit researchers and practitioners working in this area.

Richang Hong

Hefei University of Technology, China

Yahong Han

Tianjin University, China

Tat-Seng Chua

National University of Singapore, Singapore

Guest Editors 